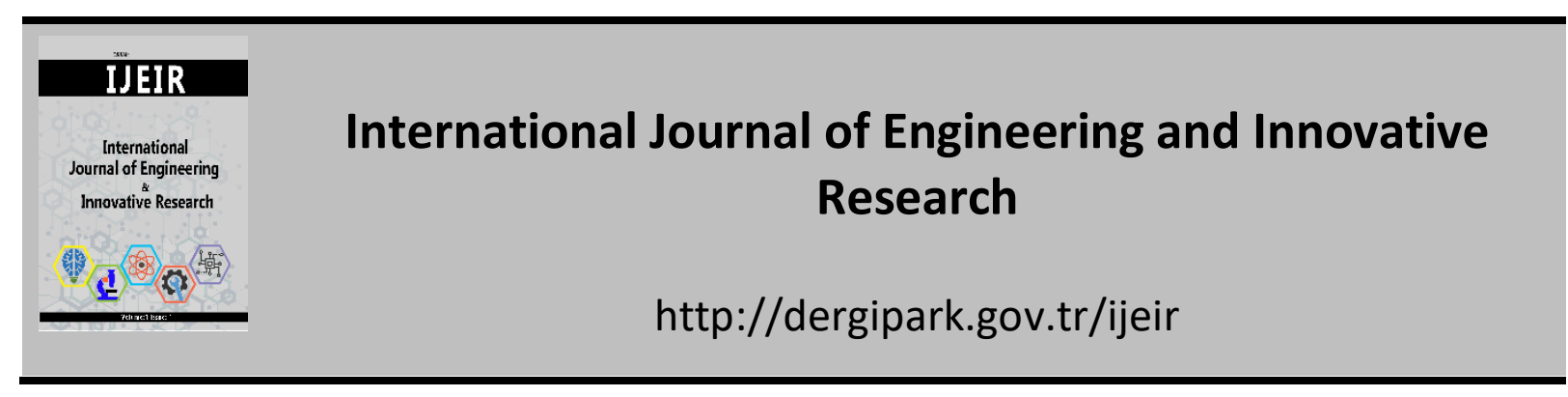

\title{
SPLIT-SPLIT PLOT ANALYSIS OF THE EFFECTS OF PROCESS PARAMETERS IN THE PRODUCTION OF POLYVINYL CHLORIDE- GRASS COMPOSITE
}

\author{
OLODU D.D ${ }^{1 *}$, ALIYEGBENOMA C.O ${ }^{2}$ () \\ ${ }^{1}$ Department of Mechanical Engineering, Faculty of Engineering, Benson Idahosa University, Benin City, Edo \\ State, NIGERIA \\ ${ }^{2}$ Department of Production Engineering University of Benin, Benin City, Edo State, NIGERIA
}

https://doi.org/10.47933/ijeir.755279

*Corresponding Author: dolodu@biu.edu.ng

(Received: 19.06.2020; Revised: 21.07.2020; Accepted: 30.07 .2020 )

\begin{abstract}
The interaction of process parameters in the production of polyvinyl chloride-grass composite poses great challenges in the polymeric industries. Most manufacturing processes of injection moulded polyvinyl chloride-grass composite products have been by trial and error due to inadequate knowledge in process parameters and their interactions. This study investigates the effects of process parameters such as percentage by volume of material, material type, barrel temperature and their interactions on the mechanical properties of the produced polyvinyl chloride-grass composite using split-split plot design. The results of the calculated Fisher's ratio (F_cal) at significant value of 0.05 for the process parameters and their interactions ranges from -855.35 to 1.00 , and were presented on ANOVA table. The results obtained shows that these process parameters contribute significantly to the production of Polyvinyl chloride-grass composite in polymeric industries.
\end{abstract}

Keywords: Mechanical properties, Polyvinyl Chloride-Grass composite, Process parameters, Split-Split Plot Design.

\section{INTRODUCTION}

A qualitative analysis of the influence of process parameters such as barrel temperature, percentage by volume of material and material type on the mechanical properties of injection moulded part will be helpful in gaining better insight into PVC-Grass composite processing methods. Moreover, inadequate investigation of the effects of the interaction of process parameters in the production of PVC-grass composites had resulted to most failure in the manufacture of these composite. The utilization of process control and process monitoring are rarely fully implemented for the production of injection moulded products. This may be due to a poor scientific understanding of the moulding process based on the complexities of the process containing multiple variables affecting the final part.

Split plot designs initially developed by Fisher in 1925 were use in agricultural experiments, and are basically the modified form of randomized block designs. These designs are used in situations where complete randomization of runs within block is not possible. These designs are used widely in industrial experiments, experiments where one set of factors may require a large amount of experimental materials (Whole Plot factors), while another set of factors 
might be applied to smaller experimental materials (Sub Plot factors) [1]. Olodu and Osarenmwinda [2] examined the effect of process parameters such as temperature in the production of polypropylene-grass composite using split-split plot experimental design, their results shows that temperature contributes significantly to the production of composites in polymeric industries. Aviles and Pinheiro [3] examined the experiments that have complete randomization order of runs which was not feasible or might be too expensive to use when performed. They concluded from their study that the use of split-plot designs and models are feasible, efficient and cheap. Goldsmith and Gaylor [1] carried out extensive investigation on optimal designs for estimating variance components in a completely random nested classification. Loeza-Serrano and Donev [4] constructed D-Optimal design for variance components estimation in a three stage crossed and nested classification. For experiments that include both crossed and nested factor in the same model, no assumption of a complete random model has been made. Ankenman et al; Aviles and Pinheiro [5,3] investigations indicates that experiments involving complete randomization of order of runs which is not feasible or too expensive to use is performed using split plot models. Chunping et al [6] carried out a study aimed to model fundamental bonding characteristics and performance of wood composite. In their work, mathematical model and a computer simulation model were developed to predict the variation of inter-element (strand) contact during mat consolidation. The mathematical predictions and the computer simulations agree well with each other. Their results showed that the relationship between the inter-element contact and the mat density was highly nonlinear and was significantly affected by the wood density and the element thickness.

This study therefore focused on the split-split plot analysis of the effects of process parameters in the production of polyvinyl chloride-grass composite.

\section{MATERIALS AND METHODS}

\subsection{Preparation and Processing of Grass}

The harvested grass was washed and soaked with dilute Sodium Hydroxide $(\mathrm{NaOH})$ of concentration $0.10 \mathrm{~mol} / \mathrm{dm}^{3}$ for 6 hours to ensure effective bonding between the grass and Polyvinyl chloride materials. The grass was ground to granules using crushing machine. The grasses were first air dried in the sun and later transferred to an oven and dried at $105^{\circ} \mathrm{C}$. It was continuously monitored until moisture content of about $4 \pm 0.2 \%$ was obtained [7]. The ground grass was screened to a particle size of $300 \mu \mathrm{m}$ diameters using vibrating sieve machine.

\subsection{Mixing, Compounding and Production of Composites}

Polyvinyl Chloride (PVC) was mixed with ground grass in the proportion of 20:80, 30:70, 40:60, 50:50, 60:40, 70:30 and 80:20 percentages by volume respectively. The prepared Polyvinyl chloride-grass composite was blended in a cylindrical container until a homogenous mixture was obtained in the composite. The homogenous mixture of the composite was feed into the hopper of injection moulding machine and were produced at various barrel temperature ranging from $210^{\circ} \mathrm{C}$ to $310^{\circ} \mathrm{C}$ respectively at an interval of $10^{\circ} \mathrm{C}$ [8]. 


\subsection{Evaluation of Polyvinyl Chloride-Grass Composite for Mechanical Strength}

The produced polyvinyl chloride-grass composite was evaluated for mechanical strength (tensile strength, proof stress, percentage elongation and flexural strength) using Equation 1 to 4 respectively [9].

Tensile strength $\quad=\frac{\text { Maximum Load }}{\text { Original Cross - Sectional Area }}$

The original cross-sectional area of the specimen is $18.9 \mathrm{~mm}^{2}$.

Proof stress $=\frac{\text { Force at yield }}{\text { Cross - Sectional Area }}$

The Cross-sectional area of specimen $=18.9 \mathrm{~mm}^{2}$

Hence, proof stress $=\frac{\text { Force at yield }}{18.9} \mathrm{~N} / \mathrm{mm}^{2}$

Percentage $(\%)$ Elongation $=\frac{\text { Extension }}{\text { Gauge Length }} \times 100 \%$

$\mathrm{EI}=\frac{\mathbf{P} \boldsymbol{L}^{3}}{\mathbf{4 8 y}}$

Where $\mathrm{y}$ is the deflection in $\mathrm{mm}, \mathrm{P}=\mathrm{Load}, \mathrm{L}=$ Length of test specimen

\subsection{The Split-Split Plot Designs}

The split-split plot design which is an experimental design was used to investigate the interaction between material type, percentage by volume of material and barrel temperature on the mechanical properties of the produced PVC-Grass composite. In simple terms, a split-split plot experiment is a blocked experiment, where the blocks themselves serve as experimental units for a subset of the factors. Analytical and numerical designs using split-split plot design was carryout to investigate the effect of process parameters in the developed PVC-Grass composite.

\subsection{The F-test}

The F-test was used for comparing the factors of the total deviation (using Equation 5). The statistical significance was tested by comparing the $\mathrm{F}$ test statistic.

$\mathrm{F}=\frac{\text { Variance between treatments }}{\text { Variance within treatments }}$

$\mathrm{F}=\frac{M S_{\text {Treatments }}}{M S_{\text {Error }}}=\frac{S S_{\text {Treatments } /(I-1)}}{S S_{\text {Error }} /(n T-1)}$

\subsection{The Interactive Model Developed for PVC-Grass Composite}

Equation 6 shows the Interactive model developed and is depicted as: 
$\mathrm{X}_{\mathrm{ijkl}}=\mu+\gamma_{i}+\beta_{j}+\delta_{l}+y_{k}+\gamma \beta_{i j}+\gamma y_{i k}+\beta y_{j k}+\gamma \delta_{i l}+\beta \delta_{j l}+y \delta_{l k}+\gamma \beta y_{i j k}+\gamma \beta \delta_{i j l}$

$+\gamma y \delta_{i k l}+\beta y \delta_{j k l}+\gamma \beta \delta y_{i j k l}+\varepsilon_{\mathrm{ijkl}}$

Where:

$\mu=$ Mean response; $\gamma_{\mathrm{I}}=$ Block variable (mechanical properties); $\beta_{\mathrm{j}}=$ Block variable (barrel temperature); $\delta_{\mathrm{l}}=$ Treatment Variable (percentage by volume of material); $y_{\mathrm{k}}=$ Treatment Variable (type of material); $\gamma \beta_{\mathrm{ij}}=$ Block interaction (mechanical properties and barrel temperature interaction); $\gamma y_{\mathrm{ik}}=$ Block and Treatment interaction (mechanical properties and type of material interaction); $\beta y_{\mathrm{jk}}=$ Treatment Interaction (barrel temperature and type of material interaction); $\gamma \delta_{\text {il }}=$ Block and Treatment interaction (mechanical properties and percentage by volume of material interaction); $\beta \delta_{\mathrm{jl}}=$ Block and Treatment interaction (barrel temperature and percentage by volume of material interaction); $y \delta_{\mathrm{lk}}=$ Treatment Interaction (percentage by volume of material and type of material interaction); $\gamma \beta y_{\mathrm{ijk}} \quad=$ Block and Treatment interaction (mechanical properties, barrel temperature and type of material interaction); $\gamma \beta \delta_{\mathrm{ijl}}=$ Block and Treatment interaction (mechanical properties, barrel temperature and Percentage by volume of material interaction); $y \gamma \delta_{\mathrm{ikl}}=$ Block and Treatment interaction (mechanical properties, type of material and Percentage by volume of material interaction) $\beta y \delta_{\mathrm{jkl}} \quad=$ Block and Treatment interaction (barrel temperature, type of material and Percentage by volume of material interaction); $\gamma \beta \delta y_{\mathrm{ijk}}=$ Block and Treatment interaction (mechanical properties, barrel temperature, type of material and percentage by volume of material interaction); $\mathrm{X}_{\mathrm{ijkl}}=$ Response Variable; $\varepsilon_{\mathrm{ijkl}}=$ Error term .

\subsection{Statistical Computations for PVC-Grass Composite}

Equation 7 to 22 was used to calculate for the sum of squares for the process parameters and their interactions which was used to investigate the effects of process parameters using splitsplit plot designs analysis. The obtained results were presented on Table 2

\section{A) Total Sum of Squares $\left(\mathrm{SS}_{\mathrm{T}}\right)$}

$\mathrm{SS}_{\mathrm{T}}=\sum_{i=1}^{I=4} \sum_{j=1}^{J=11} \sum_{k=1}^{K=2} \sum_{l=1}^{L=7} X_{i j k l}^{2}-\frac{X_{.}^{2}}{I J L K}$

Where $\mathrm{I}=4, \mathrm{~J}=11, \mathrm{~K}=2, \mathrm{~L}=7$

\section{B) Sum of squares for materials $\left(\mathbf{S S}_{\mathrm{A}}\right)$}

$\mathrm{SS}_{\mathrm{A}}=\sum_{k=1}^{K=2} \frac{X_{\ldots K}^{2}}{I J L}-\frac{X_{\ldots}^{2}}{I J L K}$

C) Sum of squares for the percentage by volume of materials $\left(\mathrm{SS}_{\mathbf{B}}\right)$

$\mathrm{SS}_{\mathrm{B}}=\sum_{l=1}^{L=7} \frac{X_{\ldots l}^{2} \ldots l}{I J K}-\frac{X^{2} \ldots}{I J L K}$

D) Sum of squares for mechanical strength $\left(\mathrm{SS}_{\mathrm{C}}\right)$

$\mathrm{SS}_{\mathrm{C}}=\sum_{i=1}^{I=4} \frac{X_{i}^{2} \ldots}{J K L}-\frac{X_{\ldots}^{2}}{I J L K}$

E) Sum of squares for temperature $\left(\mathrm{SS}_{\mathrm{D}}\right)$ 
$\mathrm{SS}_{\mathrm{D}}=\sum_{j=1}^{J=11} \frac{X_{. j}^{2} \ldots}{I K L}-\frac{X_{. \cdots}^{2}}{I J L K}$

F) (Material type) $\mathrm{X}$ (percentage by volume of material) Interaction $\left(\mathrm{SS}_{\mathrm{AB}}\right)$

$\mathrm{SS}_{\mathrm{AB}}=\sum_{k=1}^{K=2} \sum_{l=1}^{L=7} \frac{X^{2} \ldots k l}{I J}-\sum_{k=1}^{K=2} \frac{X_{\ldots k}^{2}}{I J K}-\sum_{l=1}^{L=7} \frac{X^{2} \ldots l}{I J K}+\frac{X_{\ldots}^{2} \ldots}{I J L K}$

G) (Material type) $\mathrm{X}$ (Mechanical Strength) Interaction $\left(\mathbf{S S}_{\mathrm{AC}}\right)$

$\mathrm{SS}_{\mathrm{AC}}=\sum_{i=1}^{I=4} \sum_{k=1}^{K=2} \frac{X_{i . k}^{2}}{J L}-\sum_{i=1}^{I=4} \frac{X^{2}{ }_{i} \ldots}{J K L}-\sum_{k=1}^{K=2} \frac{X_{\ldots k}^{2}}{I J L}+\frac{X_{\ldots}^{2} \ldots}{I J L K}$

H) (Material type) $\mathrm{X}$ (Temperature) Interaction $\left(\mathbf{S S}_{\mathrm{AD}}\right)$

$\mathrm{SS}_{\mathrm{AD}}=\sum_{k=1}^{K=2} \sum_{j=1}^{J=11} \frac{X^{2} \cdot j k}{I L}-\sum_{k=1}^{K=2} \frac{X^{2} \ldots k}{I J L}-\sum_{j=1}^{J=11} \frac{X_{. j}^{2} \ldots}{I K L}+\frac{X_{.2}^{2}}{I J L K}$

I) (Percentage by Volume of material) X (Mechanical Strength) Interaction (SS BC $_{\text {) }}$

$\mathrm{SS}_{\mathrm{BC}}=\sum_{i=1}^{I=4} \sum_{l=1}^{L=7} \frac{X_{i \ldots l}^{2} \ldots l}{J K}-\sum_{i=1}^{I=4} \frac{X^{2}{ }_{i} \ldots}{J K L}-\sum_{l=1}^{L=7} \frac{X^{2} \ldots l}{I J K}+\frac{X_{\ldots}^{2} \ldots}{I J L K}$

J) (Percentage by volume of material) $\mathrm{X}$ (Temperature) Interaction $\left(\mathrm{SS}_{\mathrm{BD}}\right)$

$\mathrm{SS}_{\mathrm{BD}}=\sum_{j=1}^{J=11} \sum_{l=1}^{L=7} \frac{X^{2} \cdot j \cdot l}{I K}-\sum_{j=1}^{J=11} \frac{X^{2} \cdot j \ldots}{I K L}-\sum_{l=1}^{L=7} \frac{X_{\ldots}^{2} . l}{I J K}+\frac{X_{.2}^{2}}{I J L K}$

K) (Mechanical Strength) X (Temperature) Interaction $\left(\mathbf{S S}_{\mathbf{C D}}\right)$

$\mathrm{SS}_{\mathrm{CD}}=\sum_{i=1}^{I=4} \sum_{j=1}^{J=11} \frac{X_{i j}^{2} \ldots}{K L}-\sum_{i=1}^{I=4} \frac{X^{2}{ }_{i} \ldots}{J K L}-\sum_{j=1}^{J=11} \frac{X^{2} \cdot j \ldots}{I K L}+\frac{X_{\ldots}^{2} \ldots}{I J L K}$

L) (Material type) X (Percentage by volume of material) X (Mechanical Strength) Interaction $\left(\mathbf{S S}_{\mathrm{ABC}}\right)$

$\mathrm{SS}_{\mathrm{ABC}}=\sum_{i=1}^{I=4} \sum_{k=1}^{K=2} \sum_{l=1}^{L=7} \frac{X_{i, k l}^{2}}{J}-\sum_{i=1}^{I=4} \sum_{k=1}^{K=2} \frac{X_{i, k}^{2}}{J L}-\sum_{k=1}^{K=2} \sum_{l=1}^{L=7} \frac{X^{2} . k l}{I J}+\sum_{k=1}^{K=2} \frac{X_{\ldots k}^{2} .}{I J L}$

M)(Material type) $X$ (Percentage by volume of material) $X$ (Temperature) Interaction $\left(\mathbf{S S}_{\mathrm{ABD}}\right)$

$\mathrm{SS}_{\mathrm{ABD}}=\sum_{j=1}^{J=11} \sum_{k=1}^{K=2} \sum_{l=1}^{L=7} \frac{X^{2} \cdot j k l}{I}-\sum_{j=1}^{J=11} \sum_{k=1}^{K=2} \frac{X^{2} \cdot j k}{I L}-\sum_{k=1}^{K=2} \sum_{l=1}^{L=7} \frac{X^{2} \cdot k l}{I J}+\sum_{k=1}^{K=2} \frac{X^{2} \cdot k}{I J L}$ (19)

N) (Material type) X (Mechanical strength) X (Temperature) Interaction $\left(\mathrm{SS}_{\mathrm{ACD}}\right)$ 
$\mathrm{SS}_{\mathrm{ACD}}=\sum_{i=1}^{I=4} \sum_{j=1}^{J=11} \sum_{k=1}^{K=4} \frac{X_{i j k}^{2}}{L}-\sum_{i=1}^{I=4} \sum_{j=1}^{J=11} \frac{X_{i j}^{2} \ldots}{K L}-\sum_{j=1}^{J=11} \sum_{k=1}^{K=7} \frac{X_{. j k}^{2}}{I L}+\sum_{j=1}^{J=11} \frac{X_{. j}^{2} \ldots}{I K L}$ (20)

O) (Percentage by volume of material) X (Mechanical strength) X (Temperature) Interaction $\left(\mathbf{S S}_{\mathrm{BCD}}\right)$

$\mathrm{SS}_{\mathrm{ACD}}=\sum_{i=1}^{I=4} \sum_{j=1}^{J=11} \sum_{l=1}^{L=7} \frac{X_{i j . l}^{2}}{K}-\sum_{i=1}^{I=4} \sum_{j=1}^{J=11} \frac{X_{i j}^{2} \ldots}{K L}-\sum_{j=1}^{J=11} \sum_{l=1}^{L=7} \frac{X_{. j . l}^{2}}{I K}+\sum_{j=1}^{J=11} \frac{X_{. j}^{2} \ldots}{I K L}$

P) Error Sums of Squares $S_{E}=S_{T}-S_{A}-S_{B}-S_{C}-S_{D}-S_{A B}-S S_{A C}$

$-\mathrm{SS}_{\mathrm{AD}}-\mathrm{SS}_{\mathrm{BC}}-\mathrm{SS}_{\mathrm{CD}}-\mathrm{SS}_{\mathrm{ABC}}-\mathrm{SS}_{\mathrm{ABD}}-\mathrm{SS}_{\mathrm{ACD}}$.

\subsection{Hypothesis}

The null hypothesis with its alternative were formulated for the PVC-Grass composite as follows:

Null Hypothesis $\left(\boldsymbol{H}_{\boldsymbol{o}}\right)$ : The percentage by volume of material, material type, barrel temperature and their interactions contributes significantly to the mechanical properties of the composite produced at $\alpha$-value of 0.05 .

Alternate Hypothesis $\left(\boldsymbol{H}_{1}\right)$ : The percentage by volume of material, material type, barrel temperature and their interactions does not contributes significantly to the mechanical properties of the composite produced at $\alpha$-value of 0.05 .

\section{RESULTS AND DISCUSSION}

Table 1 shows the effects of barrel temperature on mechanical properties of PVC-Grass composites while Table 2 shows ANOVA result for the effects of process parameters and their interactions on produced PVC-grass composite. Figure 1-4 shows the split-split plot analytical design of the effects of barrel temperature on tensile strength, proof stress, percentage elongation and flexural strength respectively. Figure 5-8 shows the Split-split plot numerical design of the effects of barrel temperature on tensile strength, proof stress, percentage elongation and flexural strength respectively. 
Analytical Abstract of effects of Barrel Temperature on PVC - Grass using splitsplit plot design

K=1 PVC

$\quad L=1 \quad L=2 \quad L=3 \quad L=4 \quad L=5 \quad L=6 \quad L=7$

80706050403020
K=2 GRIND

$\quad L=1 \quad L=2 \quad L=3 \quad L=4 \quad L=5 \quad L=6 \quad L=7$

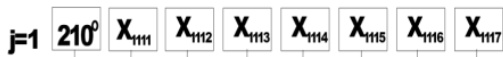

$\begin{array}{lllllllll}20 & 30 & 40 & 50 & 60 & 70 & 80\end{array}$

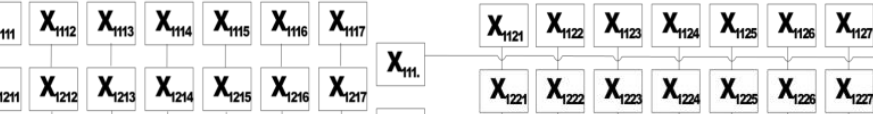

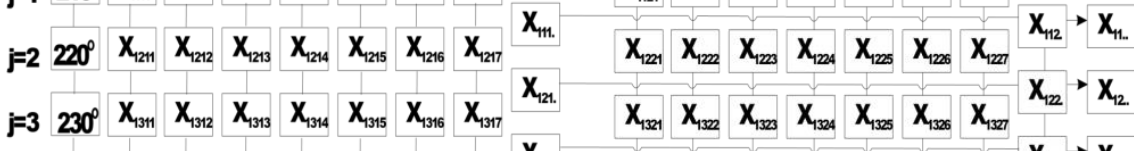

$i=1$

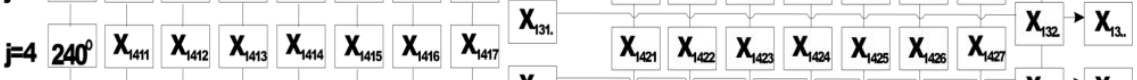

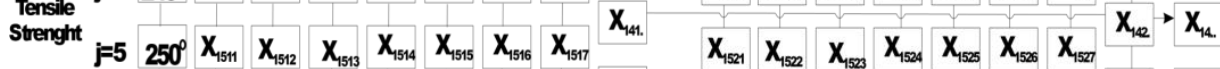

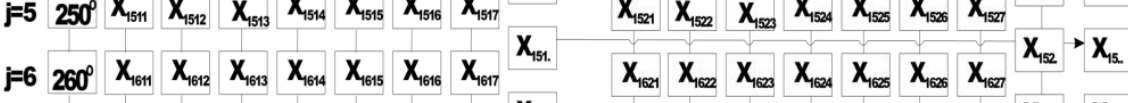

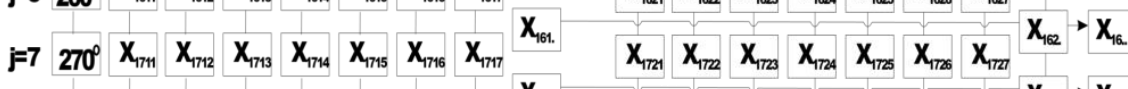

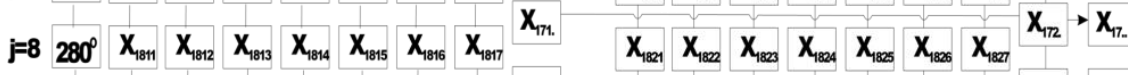

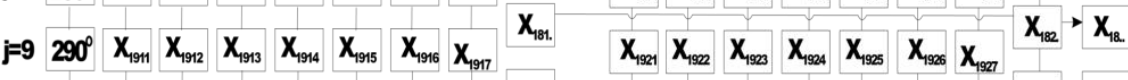

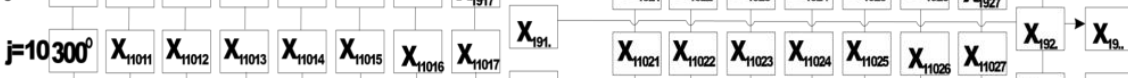

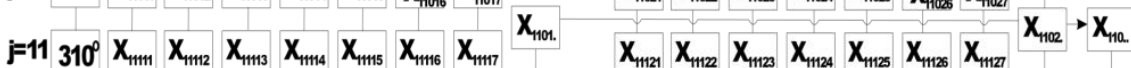

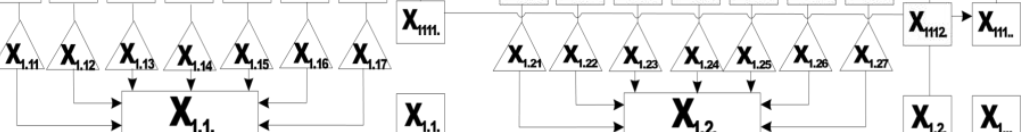

$$
\begin{aligned}
& X_{1 . . .}
\end{aligned}
$$

Figure 1. Split-split plot Analytical Design of the Effect of Barrel Temperature on Tensile Strength.

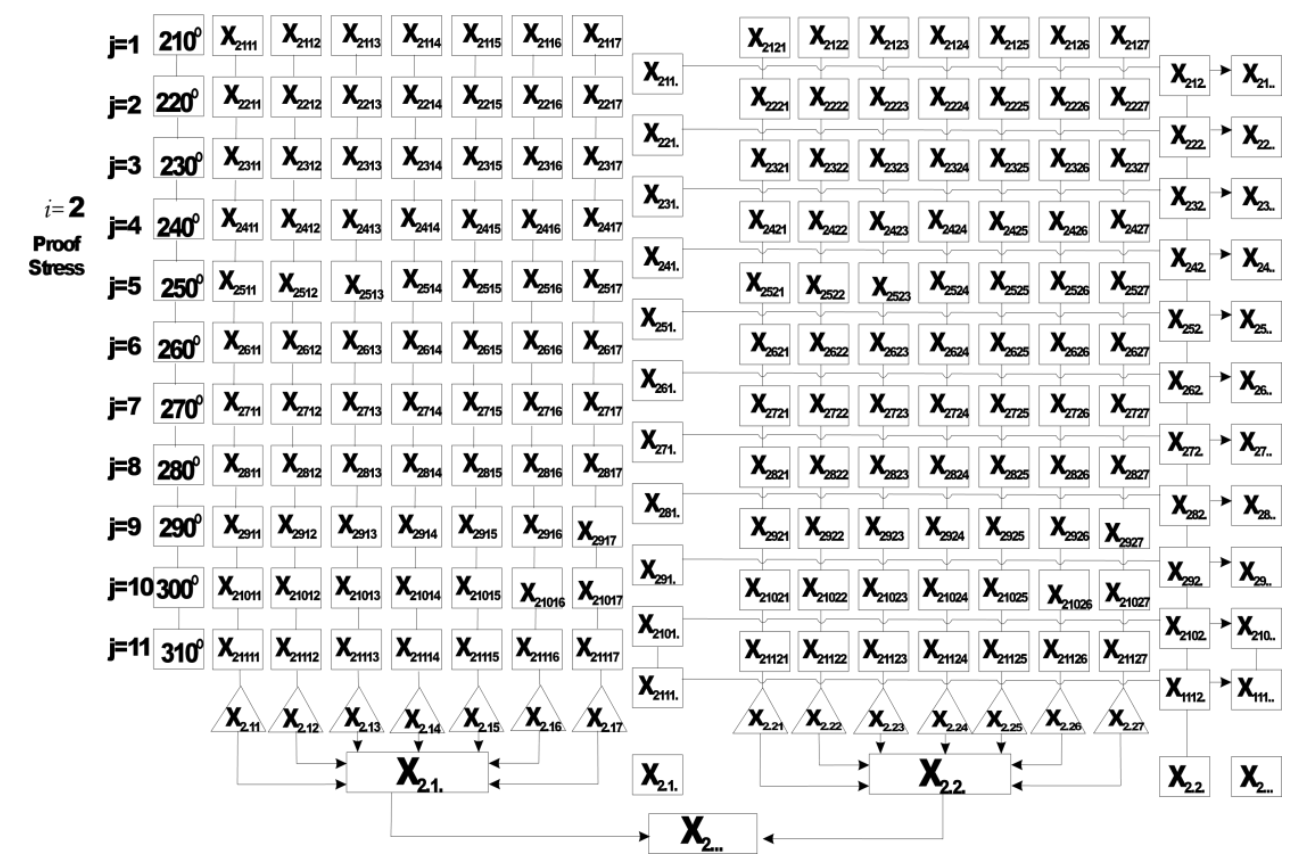

Figure 2. Split-split plot Analytical Design of the Effect of Barrel Temperature on Proof Stress. 


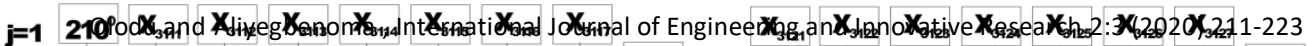

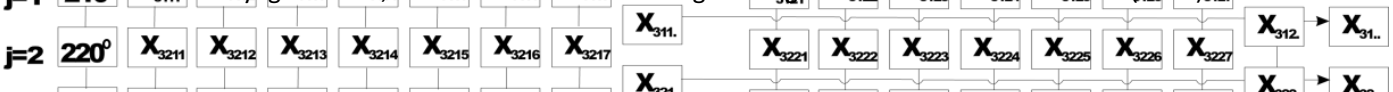

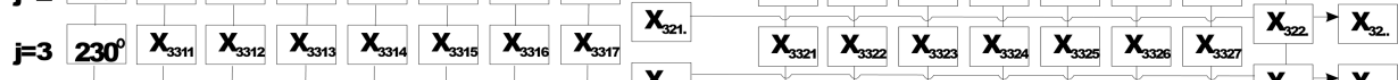

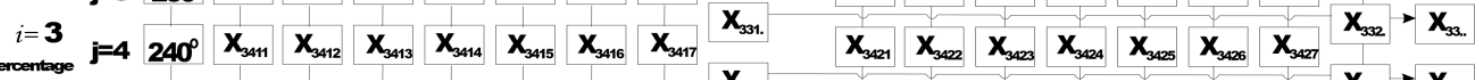

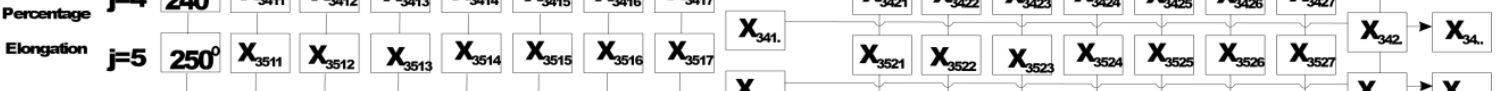

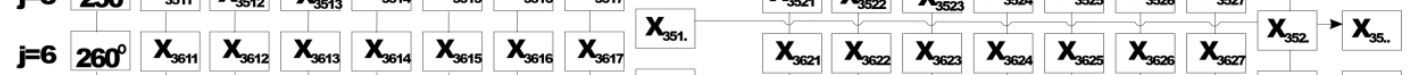

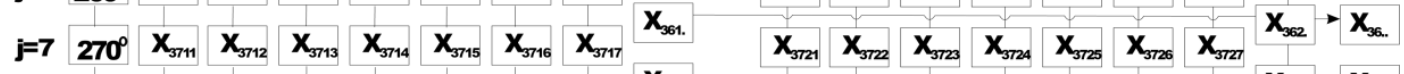

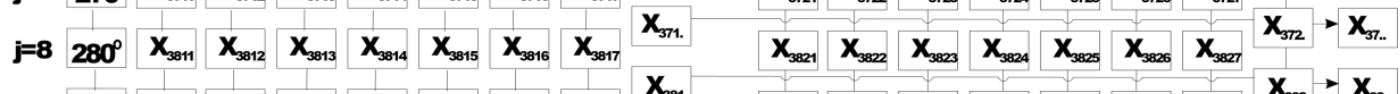

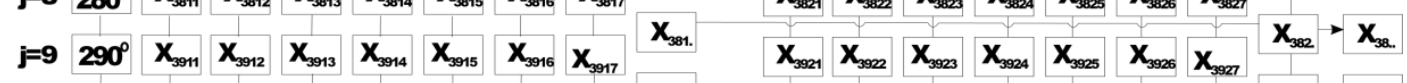

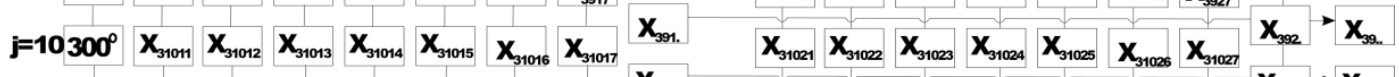

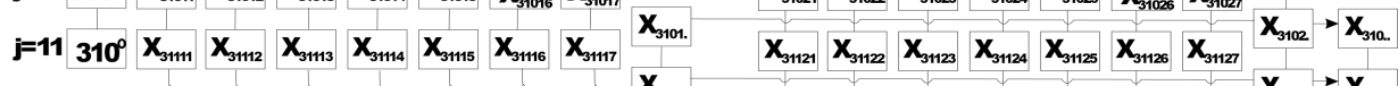
$\mathbf{x}_{311} \mathbf{x}_{312}, \mathbf{x}_{313}, \mathbf{x}_{314} \mathbf{x}_{315}, \mathbf{x}_{316} \mathbf{x}_{312}$ $\rightarrow \mathbf{x}_{3.1}$

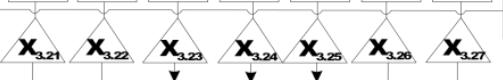

$\mathbf{X}_{31 .}$.

$\rightarrow \quad x_{3.2}^{v}$

$\mathbf{X}_{3.2} \quad \mathbf{X}_{3}$

Figure 3: Split-split plot Analytical Design of the Effect of Barrel Temperature on Percentage Elongation.

\section{Where}

\begin{tabular}{lllllllll}
$j=1$ & $210^{\circ}$ & $X_{4111}$ & $X_{4112}$ & $X_{4113}$ & $X_{4114}$ & $X_{4115}$ & $X_{4116}$ & $X_{4117}$ \\
\hline
\end{tabular}

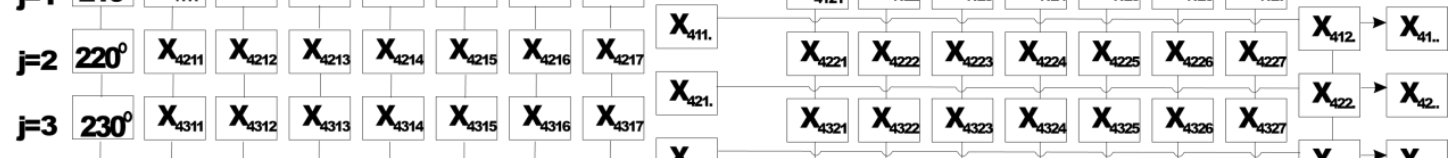

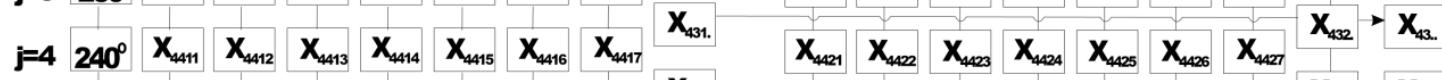

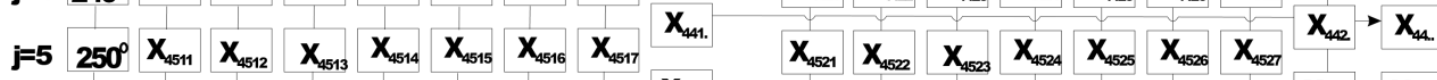

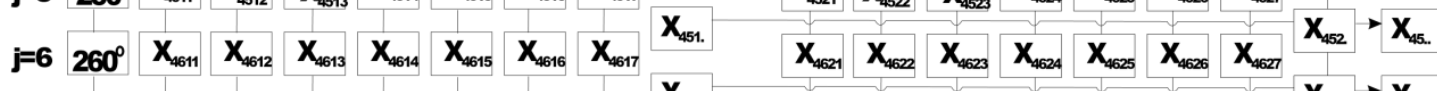

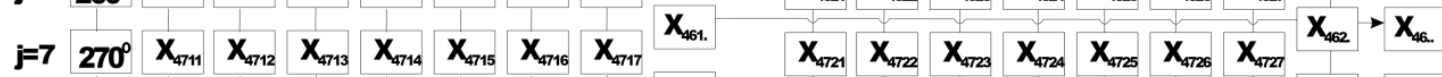

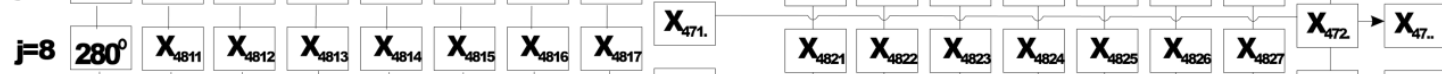
$\mathbf{j}=\mathbf{2} 290^{\circ} \mathbf{X}_{4911} \mathbf{X}_{4912} \mathbf{X}_{4913} \mathbf{X}_{4914} \mathbf{X}_{4915} \mathbf{X}_{4916} \mathbf{X}_{4917} \mathbf{X}_{481 .} \quad \mathbf{X}_{4921} \mathbf{X}_{4922} \mathbf{X}_{4923} \mathbf{X}_{4924} \mathbf{X}_{4925} \mathbf{X}_{4926} \mathbf{X}_{4927} \mathbf{X}_{482} \rightarrow \mathbf{X}_{48.1}$

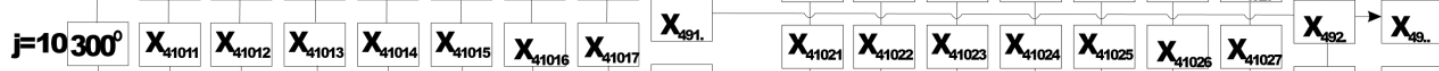
$j=11310^{\circ} \mathbf{X}_{4111} \mathbf{X}_{41112} \mathbf{X}_{41113} \mathbf{X}_{41114} \mathbf{X}_{41115} \mathbf{X}_{41116} \mathbf{X}_{41177} \mathbf{X}_{4101 .} \quad \mathbf{X}_{41121} \mathbf{X}_{41122} \mathbf{X}_{41123} \mathbf{X}_{41124} \mathbf{X}_{41125} \mathbf{X}_{41126} \mathbf{X}_{41127} \mathbf{X}_{4102} \rightarrow \mathbf{X}_{410 .}$

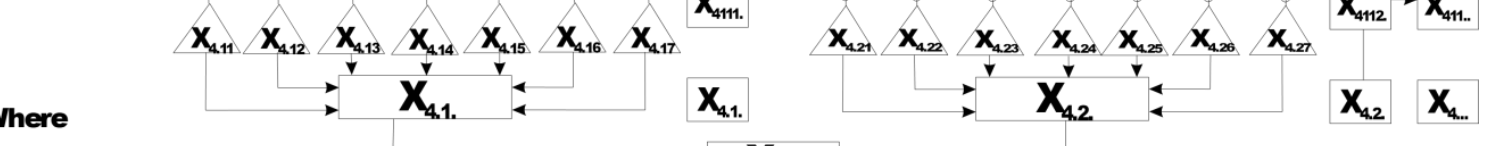

i = Mechanical Properties

j = Temperature

$k=$ Type of Material

I = Percentage by volume of material

Figure 4. Split-split Plot Analytical Design of the Effect of Barrel Temperature on Flexural Strength. 


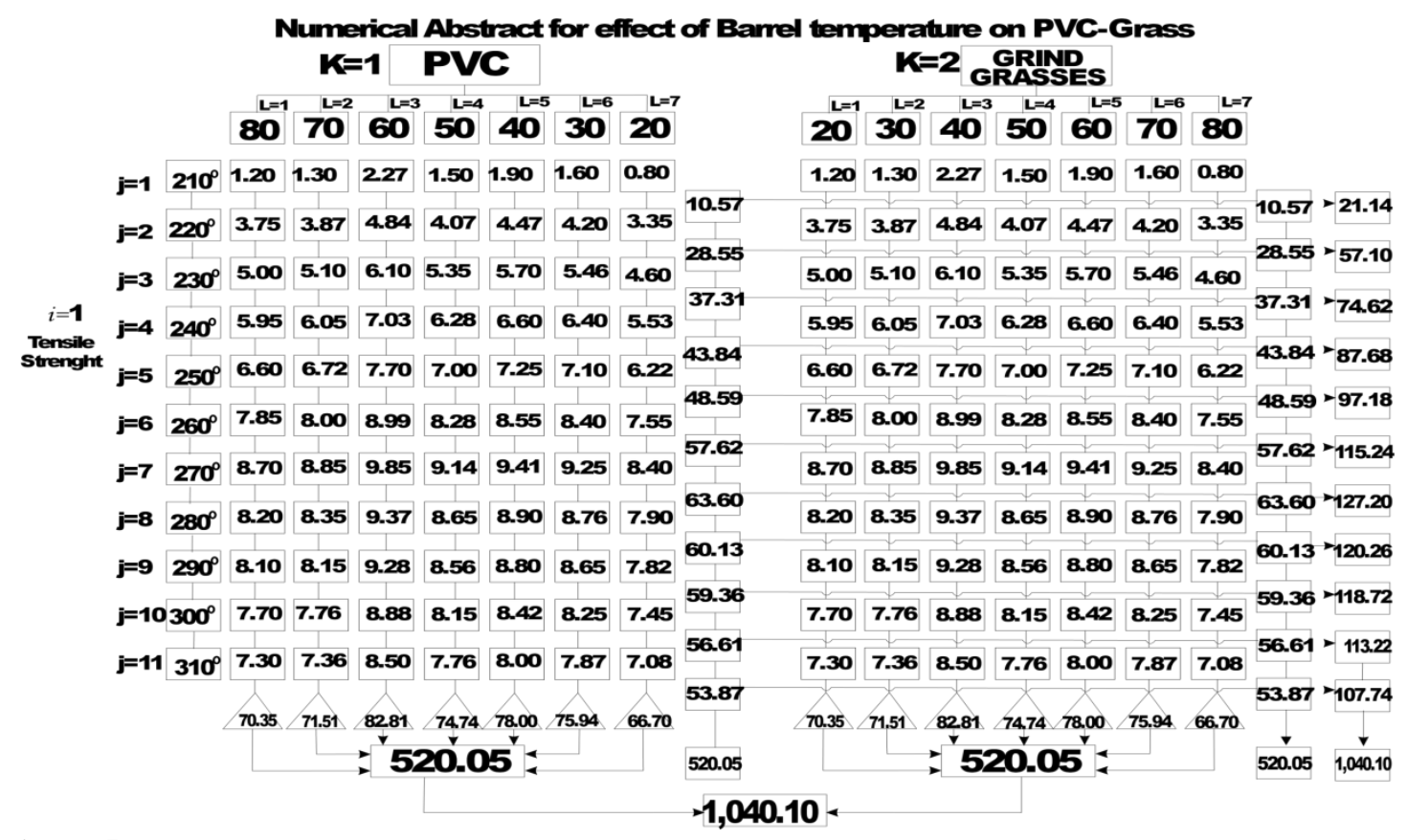

Figure 5: Split-split plot Numerical Design of the Effect of Barrel Temperature on Tensile Strength.

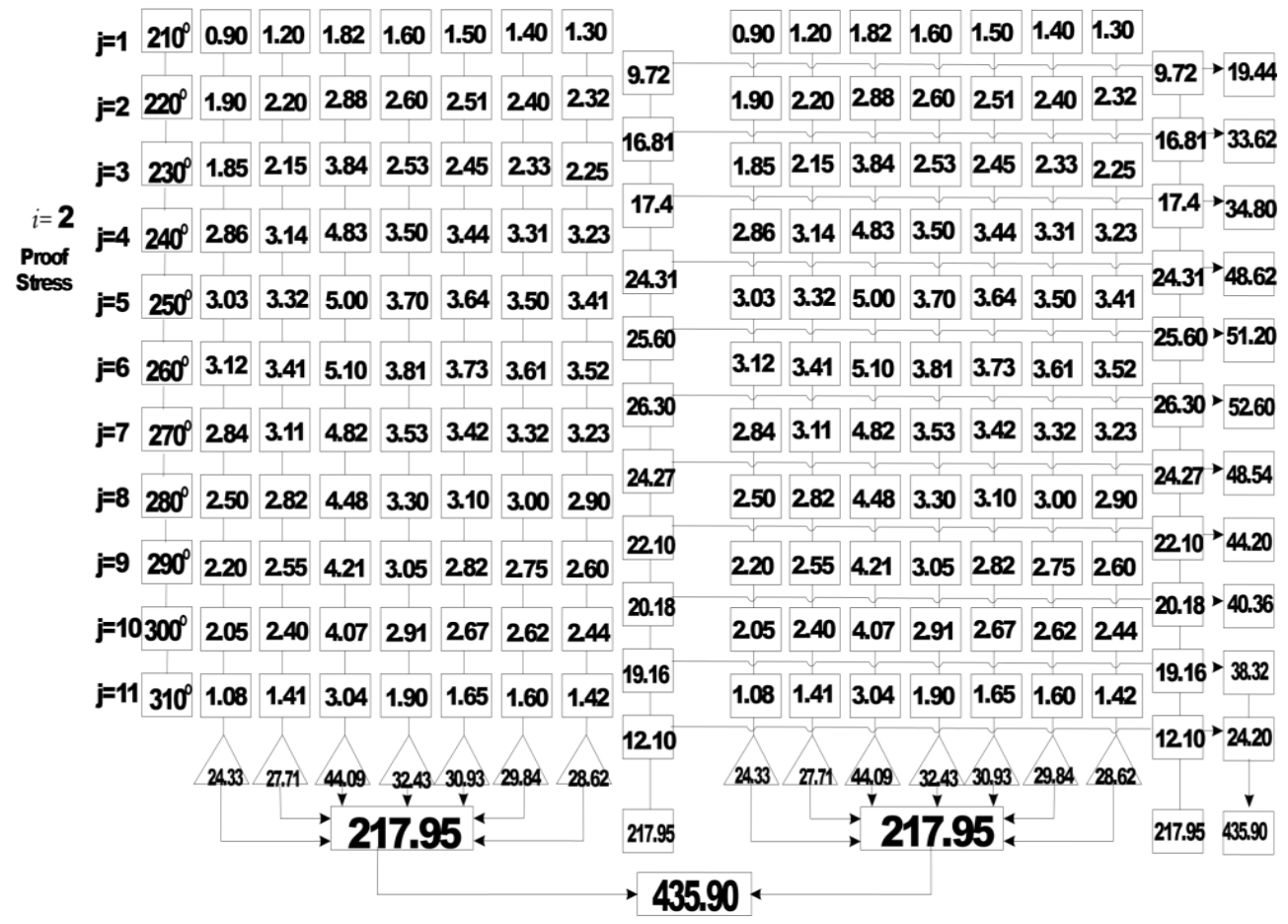

Figure 6: Split-split plot Numerical Design of the Effect of Barrel Temperature on Proof Stress. 


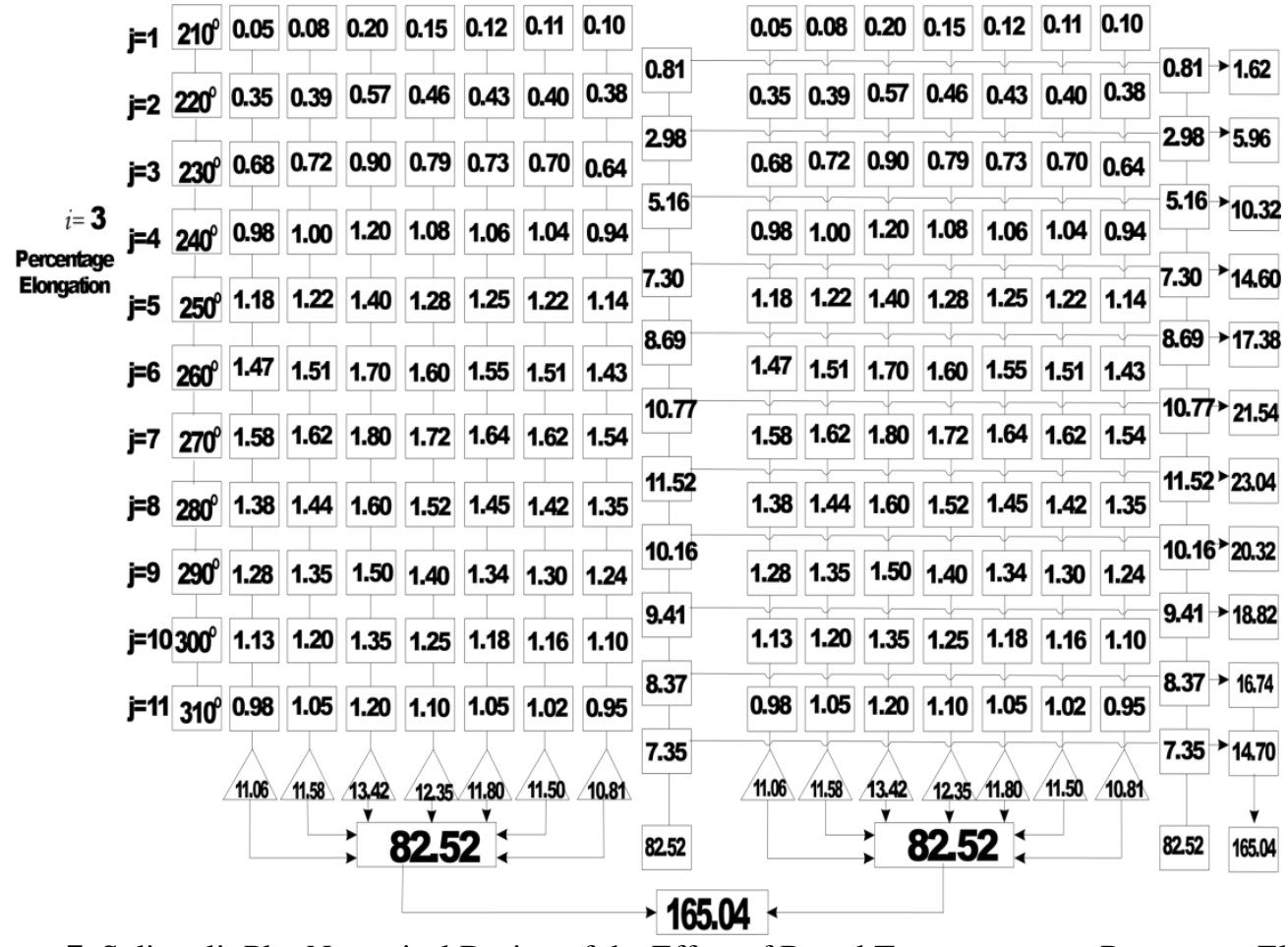

Figure 7. Split-split Plot Numerical Design of the Effect of Barrel Temperature on Percentage Elongation.

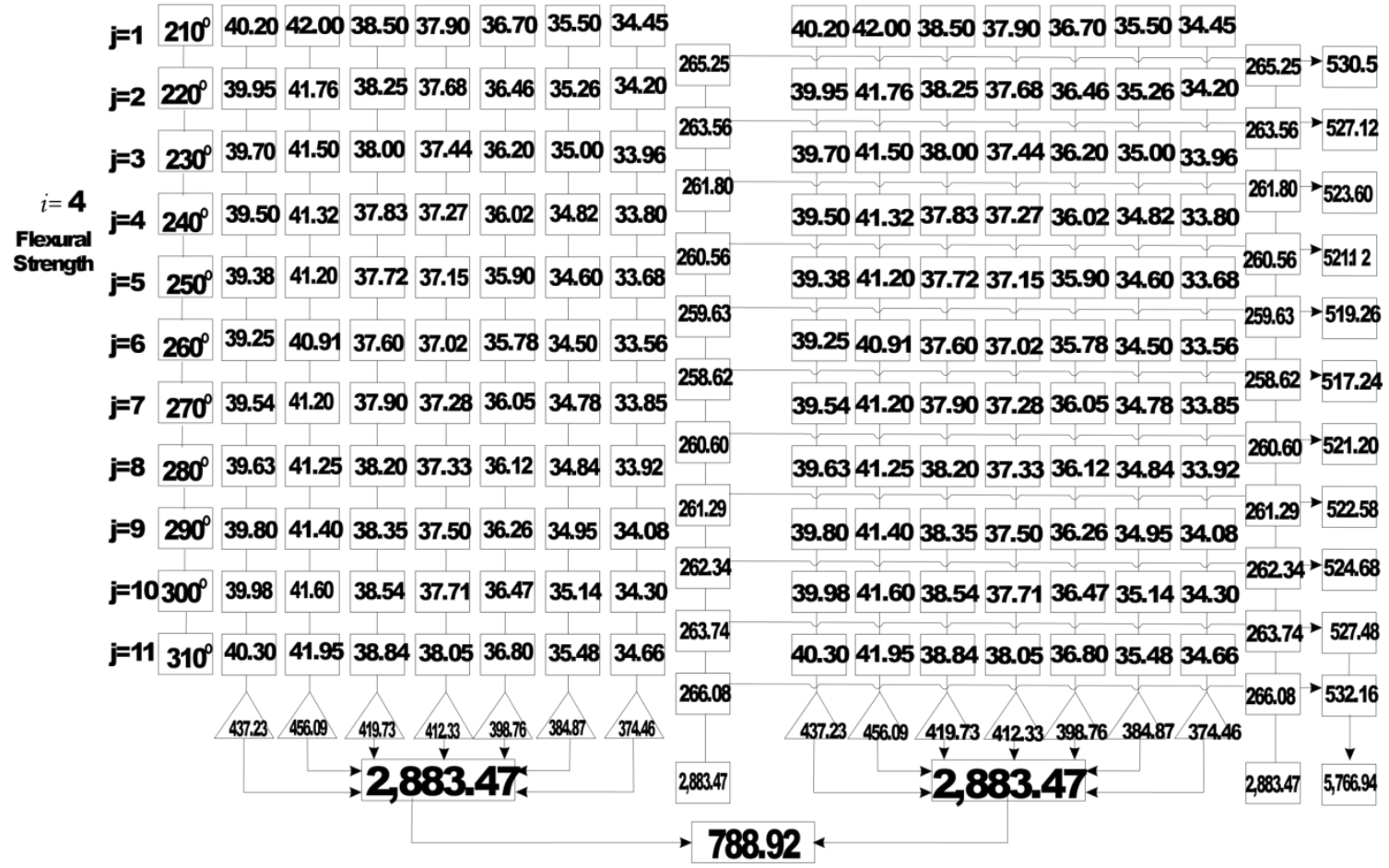

Figure 8. Split-split Plot Numerical Design of the Effect of Barrel Temperature on Flexural Strength. 
Table 1. Effects of Barrel Temperature on Mechanical Properties of PVC-Grass Composites.

\begin{tabular}{|c|c|c|c|c|c|c|c|c|c|c|c|c|c|}
\hline \multirow[b]{2}{*}{$\begin{array}{l}\text { MECHANICAL } \\
\text { PROPERTY }\end{array}$} & \multirow[b]{2}{*}{$\begin{array}{c}\text { PERCENTAGE } \\
\text { BY VOLUME OF } \\
\text { PVC } \\
\text { (M) }\end{array}$} & \multirow[b]{2}{*}{$\begin{array}{l}\text { PERCENTAGE BY } \\
\text { VOLUME OF } \\
\text { GRASS } \quad \text { (K) }\end{array}$} & \multicolumn{11}{|c|}{ TEMPERATURE $\left({ }^{\circ} \mathrm{C}\right)$} \\
\hline & & & 210 & 220 & 230 & 240 & 250 & 260 & 270 & 280 & 290 & 300 & 310 \\
\hline \multirow{7}{*}{$\begin{array}{c}\text { TENSILE } \\
\text { STRENGTH } \\
\left(\mathrm{N} / \mathrm{mm}^{2}\right)\end{array}$} & 80 & 20 & 1.20 & 3.75 & 5.00 & 5.95 & 6.60 & 7.85 & 8.70 & 8.20 & 8.10 & 7.70 & 7.30 \\
\hline & 70 & 30 & 1.30 & 3.87 & 5.10 & 6.05 & 6.72 & 8.00 & 8.85 & 8.35 & 8.15 & 7.76 & 7.36 \\
\hline & 60 & 40 & 2.27 & 4.84 & 6.10 & 7.03 & 7.70 & 8.99 & 9.85 & 9.37 & 9.28 & 8.88 & 8.50 \\
\hline & 50 & 50 & 1.50 & 4.07 & 5.35 & 6.28 & 7.00 & 8.28 & 9.14 & 8.65 & 8.56 & 8.15 & 7.76 \\
\hline & 40 & 60 & 1.90 & 4.47 & 5.70 & 6.60 & 7.25 & 8.55 & 9.41 & 8.90 & 8.80 & 8.42 & 8.00 \\
\hline & 30 & 70 & 1.60 & 4.20 & 5.46 & 6.40 & 7.10 & 8.40 & 9.25 & 8.76 & 8.65 & 8.25 & 7.87 \\
\hline & 20 & 80 & 0.80 & 3.35 & 4.60 & 5.53 & 6.22 & 7.55 & 8.40 & 7.90 & 7.82 & 7.45 & 7.08 \\
\hline \multirow{7}{*}{$\begin{array}{l}\text { PROOF STRESS } \\
\left(\mathrm{N} / \mathrm{mm}^{2}\right)\end{array}$} & 80 & 20 & 0.90 & 1.90 & 1.85 & 2.86 & 3.03 & 3.12 & 2.84 & 2.50 & 2.20 & 2.05 & 1.08 \\
\hline & 70 & 30 & 1.20 & 2.20 & 2.15 & 3.14 & 3.32 & 3.41 & 3.11 & 2.82 & 2.55 & 2.40 & 1.41 \\
\hline & 60 & 40 & 1.82 & 2.88 & 3.84 & 4.83 & 5.00 & 5.10 & 4.82 & 4.48 & 4.21 & 4.07 & 3.04 \\
\hline & 50 & 50 & 1.60 & 2.60 & 2.53 & 3.50 & 3.70 & 3.81 & 3.53 & 3.30 & 3.05 & 2.91 & 1.90 \\
\hline & 40 & 60 & 1.50 & 2.51 & 2.45 & 3.44 & 3.64 & 3.73 & $\begin{array}{l}3.42 \\
3.32\end{array}$ & 3.10 & 2.82 & 2.67 & 1.65 \\
\hline & 30 & 70 & 1.40 & 2.40 & 2.33 & 3.31 & 3.50 & 3.61 & 3.32 & 3.00 & 2.75 & 2.62 & 1.60 \\
\hline & 20 & 80 & 1.30 & 2.32 & 2.25 & 3.23 & 3.41 & 3.52 & & 2.90 & 2.60 & 2.44 & 1.42 \\
\hline \multirow{7}{*}{$\begin{array}{c}\text { PERCENTAGE } \\
\text { ELONGATION } \\
(\%)\end{array}$} & 80 & 20 & 0.05 & 0.35 & 0.68 & 0.98 & 1.18 & 1.47 & 1.58 & 1.38 & 1.28 & 1.13 & 0.98 \\
\hline & 70 & 30 & 0.08 & 0.39 & 0.72 & 1.00 & 1.22 & 1.51 & 1.62 & 1.44 & 1.35 & 1.20 & 1.05 \\
\hline & 60 & 40 & 0.20 & 0.57 & 0.90 & 1.20 & 1.40 & 1.70 & 1.80 & 1.60 & 1.50 & 1.35 & 1.20 \\
\hline & 50 & 50 & 0.15 & 0.46 & 0.79 & 1.08 & 1.28 & 1.60 & 1.72 & 1.52 & 1.40 & 1.25 & 1.10 \\
\hline & 40 & 60 & 0.12 & 0.43 & 0.73 & 1.06 & 1.25 & 1.55 & 1.64 & 1.45 & 1.34 & 1.18 & 1.05 \\
\hline & 30 & 70 & 0.11 & 0.40 & 0.70 & 1.04 & 1.22 & 1.51 & 1.62 & 1.42 & 1.30 & 1.16 & 1.02 \\
\hline & 20 & 80 & 0.10 & 0.38 & 0.64 & 0.94 & 1.14 & 1.43 & 1.54 & 1.35 & 1.24 & 1.10 & 0.95 \\
\hline \multirow{7}{*}{$\begin{array}{l}\text { AVERAGE } \\
\text { DEFLECTION } \\
(\mathrm{mm})\end{array}$} & 80 & 20 & 8.70 & 8.80 & 8.65 & 8.70 & 8.67 & 8.60 & 8.87 & 8.67 & 8.88 & 8.67 & 8.64 \\
\hline & 70 & 30 & 9.87 & 9.64 & 9.37 & 9.21 & 9.11 & 9.00 & 9.08 & 9.15 & 9.31 & 9.44 & 9.66 \\
\hline & 60 & 40 & 8.11 & 7.95 & 7.77 & 7.66 & 7.59 & 7.52 & 7.57 & 7.62 & 7.74 & 7.83 & 7.68 \\
\hline & 50 & 50 & 8.31 & 8.34 & 7.95 & 7.83 & 7.77 & 7.68 & 7.74 & 7.79 & 7.90 & 7.99 & 8.14 \\
\hline & 40 & 60 & 8.64 & 8.47 & 8.26 & 8.13 & 8.09 & 8.01 & 8.10 & 8.14 & 8.26 & 8.35 & 8.21 \\
\hline & 30 & 70 & 8.89 & 8.69 & 8.48 & 8.37 & 8.30 & 8.21 & 8.29 & 8.36 & 8.48 & 8.58 & 8.41 \\
\hline & 20 & 80 & $9 . .08$ & 8.89 & 8.65 & 8.52 & 8.44 & 8.34 & 8.44 & 8.51 & 8.65 & 8.75 & 8.93 \\
\hline \multirow{7}{*}{$\begin{array}{c}\text { FLEXURAL } \\
\text { STRENGTH } \\
\times 10^{3}\left(\mathrm{~N} / \mathrm{mm}^{2}\right)\end{array}$} & 80 & 20 & 18.80 & 19.32 & 19.93 & 20.30 & 20.50 & 20.78 & 20.58 & 20.30 & 19.92 & 19.63 & 20.10 \\
\hline & 70 & 30 & 20.70 & 21.20 & 21.81 & 22.20 & 22.43 & 22.71 & 22.51 & 22.33 & 21.95 & 21.64 & 21.16 \\
\hline & 60 & 40 & 25.20 & 25.70 & 26.30 & 26.70 & 26.92 & 27.20 & 27.00 & 26.82 & 26.40 & 26.10 & 26.60 \\
\hline & 50 & 50 & 24.60 & 25.10 & 25.70 & 26.10 & 26.31 & 26.60 & 26.41 & 26.23 & 25.87 & 25.58 & 25.10 \\
\hline & 40 & 60 & 23.65 & 24.13 & 24.73 & 25.15 & 25.25 & 25.53 & 25.24 & 25.10 & 24.73 & 24.47 & 24.90 \\
\hline & 30 & 70 & 23.00 & 23.51 & 24.11 & 24.41 & 24.62 & 24.91 & 24.64 & 24.46 & 24.10 & 23.82 & 24.30 \\
\hline & 20 & 80 & 22.50 & 23.00 & 23.62 & 24.00 & 24.22 & 24.51 & 24.21 & 24.02 & 23.64 & 23.36 & 22.90 \\
\hline
\end{tabular}

Table 2. Anova Result Table for Effects of Barrel Temperature on PVC-Grass Composite.

\begin{tabular}{|c|c|c|c|c|c|}
\hline $\begin{array}{l}\text { Sources of } \\
\text { Variation }\end{array}$ & $\begin{array}{c}\text { Sum of } \\
\text { Squares (SS) }\end{array}$ & Degree of freedom & $\begin{array}{c}\text { Mean of } \\
\text { Squares (MS) }\end{array}$ & $\begin{array}{c}\begin{array}{c}\text { Fisher's Ratio } F_{\text {cal }} \\
\alpha=0.05\end{array} \\
\end{array}$ & $\begin{array}{c}\text { Fisher's Ratio } \\
\mathrm{F}_{\text {Table }} \\
\end{array}$ \\
\hline $\mathbf{S S}_{\mathrm{A}}$ & 0.00 & $K-1=1$ & 0.00 & 0.00 & 5.99 \\
\hline $\mathrm{SS}_{\mathrm{B}}$ & 331.30 & $L-1=6$ & 55.22 & 0.00 & 4.28 \\
\hline $\mathrm{SS}_{\mathrm{c}}$ & $50,049.04$ & $1-1=3$ & 16683.01 & 0.00 & 9.28 \\
\hline $\mathrm{SS}_{\mathrm{D}}$ & 522.56 & $J-1=10$ & 52.28 & 0.00 & 2.98 \\
\hline $\mathrm{SS}_{\mathrm{AB}}$ & -0.00001 & $(K-1)(L-1)=6$ & $-1.67 \times 10^{-6}$ & 0.00 & 8.94 \\
\hline $\mathbf{S S}_{\mathrm{AC}}$ & -0.000018 & $(K-1)(I-1)=3$ & $-1.60 \times 10^{-6}$ & 0.00 & 3.16 \\
\hline $\mathrm{SS}_{\mathrm{AD}}$ & 0.00 & $(\mathrm{~K}-1)(\mathrm{J}-1)=10$ & 0.00 & 0.00 & 1.99 \\
\hline $\mathrm{SS}_{\mathrm{BC}}$ & 406.65 & $(L-1)(I-1)=18$ & 22.59 & 1.00 & 2.01 \\
\hline $\mathrm{SS}_{\mathrm{BD}}$ & -7697.86 & $(\mathrm{~L}-1)(\mathrm{J}-1)=60$ & 128.30 & -855.35 & 0.51 \\
\hline $\mathbf{S S}_{\mathrm{CD}}$ & 392.25 & $(I-1)(J-1)=30$ & 13.07 & 0.00 & 1.37 \\
\hline $\mathrm{SS}_{\mathrm{ABC}}$ & 406.65 & $(K-1)(L-1)(I-1)=18$ & 22.59 & 0.43 & 2.98 \\
\hline $\mathbf{S S}_{\mathrm{ABD}}$ & -9.03 & $(K-1)(L-1)(I-1)=60$ & -0.15 & -0.01 & 0.17 \\
\hline $\mathrm{SS}_{\mathrm{ACD}}$ & -0.000027 & $(\mathrm{~K}-1)(\mathrm{I}-1)(\mathrm{J}-1)=30$ & -0.00 & 0.00 & 1.93 \\
\hline $\mathrm{SS}_{\mathrm{BCD}}$ & 8070.08 & $(\mathrm{~L}-1)(\mathrm{I}-1)(\mathrm{J}-1)=180$ & 44.83 & -135.85 & 6.57 \\
\hline $\mathbf{S S}_{\mathrm{E}}$ & -60.27 & $(\mathrm{I}-1)(\mathrm{J}-1)(\mathrm{K}-1)(\mathrm{L}-1)=180$ & -0.33 & & \\
\hline $\mathbf{S S}_{\mathrm{T}}$ & 51707.85 & IJKL-1=615 & & & \\
\hline
\end{tabular}

\subsection{Interpretation of the Results}

Figure 1-4 shows the split-split plot analytical design of the effects of barrel temperature on tensile strength, proof stress, percentage elongation and flexural strength respectively. The values in Table 1 was input into the analytical split-split plot design which resulted to the 
Split-split plot numerical design of the effects of barrel temperature on tensile strength, proof stress, percentage elongation and flexural strength respectively (Figure 5-8). Equation 7 to 22 was used to evaluate for the sum of squares for the process parameters and their interactions, the values obtained were presented on Table 2 .

The investigation of treatment effect of materials $\left(\mathrm{SS}_{\mathrm{A}}\right)$, percentage by volume of materials $\left(\mathrm{SS}_{\mathrm{B}}\right)$, mechanical strength $\left(\mathrm{SS}_{\mathrm{C}}\right)$ and barrel temperature $\left(\mathrm{SS}_{\mathrm{D}}\right)$ respectively shows that the calculated Fisher's ratio values were less than the Fisher ratio values obtained from the table at $\alpha$-value of 0.05 (Table 2). The results compared favourably with the results obtained by Goos, and Vandebroek [10] using D-optimal Split-Plot Designs with given numbers and sizes of whole plots. From the results obtained, it shows that the experimental data do not furnish enough evidence to reject the null hypothesis $H_{o}$ treatment at $\alpha$-value of 0.05 . This shows that the treatment effect and the block effect of process parameters contribute significantly to the mechanical property of the produced PVC-Grass composite in industries.

Furthermore, the interaction of the process parameters obtained from treatment effect such as Material type and percentage by volume of material Interaction $\left(\mathrm{SS}_{\mathrm{AB}}\right)$; Material type and Mechanical Strength Interaction $\left(\mathrm{SS}_{\mathrm{AC}}\right)$; Material type and Temperature Interaction $\left(\mathrm{SS}_{\mathrm{AD}}\right)$; Percentage by Volume of material and Mechanical Strength Interaction $\left(\mathrm{SS}_{\mathrm{BC}}\right)$; Percentage by volume of material and Temperature Interaction $\left(\mathrm{SS}_{\mathrm{BD}}\right)$; Mechanical Strength and Temperature Interaction $\left(\mathrm{SS}_{\mathrm{CD}}\right)$; Material type, Percentage by volume of material and Temperature Interaction ( $\mathrm{SS}_{\mathrm{ABD}}$ ); Material type, Mechanical strength and Temperature Interaction $\left(\mathrm{SS}_{\mathrm{ACD}}\right)$; Percentage by volume of material, Mechanical strength and Temperature Interaction $\left(\mathrm{SS}_{\mathrm{BCD}}\right)$; Material type, Percentage by volume of material and Mechanical Strength Interaction $\left(\mathrm{SS}_{\mathrm{ABC}}\right)$ respectively shows that the calculated Fisher's ratio value is less than the Fisher ratio obtained from the table at $\alpha$-value of 0.05 (Table 2). The results compare favourably with the results obtained by Goos and Vandebroek[10]; Loeza and Donev [4]; Edelugo [12]. The experimental data do not furnish enough evidence to reject the null hypothesis $H_{o}$ treatment at $\alpha$-value of 0.05 . This shows that the treatment effect and the block effect interaction of these process parameters contribute significantly to the mechanical property of the produced PVC-Grass composite in industries.

\section{CONCLUSION}

The results obtained from the interactive model developed using the split-split plot design indicates that there were strong interaction between barrel temperature, type of material and percentage by volume of material on mechanical properties (Tensile Strength, Proof Stress, Percentage Elongation and Flexural Strength) for the produced PVC-Grass composites. Hence, these process parameters contributes significantly to the produced injection moulded PVC-Grass composite. Decisions made based on the hypothesis statements shows that there were no enough evidence to reject the null hypothesis at $\alpha$-value of 0.05 for PVC-Grass composite. The developed interactive model will also be useful to researcher, industrialist and small scale manufacturer to ease the production of plastic-grass composite in polymeric industries. 


\section{REFERENCES}

[1] Goldsmith, CH; Gaylor, DW. Three stage nested designs for estimating variance components. Journal of Technometrics, vol.12, pp.487-498, 1970.

[2] Olodu, D.D; Osarenmwinda, J.O. Effects of Process Parameters in the Production of High Density Polyethylene-Grass Composite. Journal of Applied Sciences and Environmental management, Vol. 22, No 9, page 1479-1483, 2018.

[3] Avilles, A.I; Pinheiro, J.C. Optimal Design for Mixed Effects Models with two Random Nested Factors. Journal of Statistical Sinica, vol.13, pp.385-401, 2015.

[4] Loeza, S; Donev, A.N. Construction of Experimental Design to estimate Variance Components. Journal of Computational Statistics and Data Analysis, vol.71, pp.1168-1177, 2014.

[5] Ankenman, B.E; Liu, H; Karr, AF; Picka, J.D. A Class of Experimental Designs for Estimating a Response Surface and Variance Components. Journal of Technometrics, vol.44, pp.45-54, 2001.

[6] Chunping, D; Changing. Y; Cheng, Z. Theoretical Modeling of Bonding Characteristics and Performance of Wood Composites of Inter-element Contact. Journal of Wood and Fiber Science, vol.39, pp.48-55, 2007.

[7] Adeyemi, S.O; Adeyemi, M.B. Temperature Effect on Extrusion Moulded Plastic. International Journal of Materials and Product Technology, vol. 5, No 6, pp.300-311, 2016.

[8] Olodu, D.D. Modelling and Development of Injection Moulded Plastic-Grass Composites. PhD Thesis, Department of Production Engineering, Faculty of Engineering,University of Benin, Nigeria, 2017.

[9] Osaremwinda, J.O; Olodu, D.D. Effects of Barrel Temperature on the Mechanical Properties of Injection Moulded Product. .Nigeria Journal of Technology (NIJOTECH), Vol. 34, No.2, pp.292-296, 2015.

[10] Goos, P; Vandebroek, M. D-optimal Split-Plot Designs with Given Numbers and Sizes of Whole Plots. Journal of Technometrics, vol.45, pp.235-245, 2003

[11] Harless, TE; Wagner, PH; Short, RD; Seale, PH; Mitchell, D. A model to Predict the Density Profile of Particleboard. Journal of Wood and Fiber Science, vol.19, pp81- 92, 1987.

[12] Edelugo, S.O. Effects of Reinforcements Combination on the Mechanical Strength of Glass Reinforced Plastic under Increased Temperature Conditions. Nigeria Journal of Technology, vol.23, No. 1, pp.39-47, 2004. 